\title{
Etiology of acute diarrhea in patients requiring hospitalization in Clinical Infectious Diseases Hospital - Constanța
}

\author{
Anca Dumitrescu', Sorina Carp ${ }^{1}$, Maria Margareta llie ${ }^{1}$, Elena Dumea ${ }^{1,2}$, Sorin Rugină ${ }^{1,2}$, Stela Halichidis ${ }^{1,2}$, \\ Simona Claudia Cambrea ${ }^{1,2^{*}}$ \\ From The 10th Edition of the Scientific Days of the National Institute for Infectious Diseases "Prof Dr Matei \\ Bals" \\ Bucharest, Romania. 15-17 October 2014
}

\section{Background}

Acute diarrhea (AD) plays an important role in human pathology because of its high incidence, especially in children. The study aims to evaluate the etiology of $\mathrm{AD}$ and bacterial isolated drug resistance for hospitalized patients during July 2013 and June 2014.

\section{Methods}

During July 2013 and June 2014 stool cultures for the identification of the bacterial pathogen agents were performed and we used latex agglutination tests for the detection of rotavirus for patients who presented at the Infectious Diseases Hospital with AD.

\section{Results}

In the study period 3,929 patients with AD (with 353 positive cases) presented to the Infectious Diseases Hospital out of which 2,550 children (with 301 positive cases) and 1,371 adults (with 52 positive cases). In children rotavirus enteritis ranked first in the etiology of infectious enteritis with a confirmed etiology of $62 \%$ over the entire period (186 cases) with a peak incidence in January-March - 78\% of enteritis with infectious etiology confirmed (92 cases). Of bacterial causes of enteritis in children under 2 years, Klebsiella ranked first with $60 \%$ (32 cases), followed by enteropathogenic E. coli, with $25.9 \%$ ( 14 cases). The isolated strains of Klebsiella were $100 \%$ sensitive to imipenem, $92 \%$ sensitive to quinolones, $80 \%$ sensitive to ceftriaxone but only

* Correspondence: claudia_cambrea@hotmail.com

${ }^{1}$ Clinical Infectious Diseases Hospital of Constanța, Romania

Full list of author information is available at the end of the article
$33.3 \%$ sensitive to amoxicillin/clavulanic acid. In children over 2 years, the most frequently encountered bacteria was Salmonella spp. $-47.5 \%$ of bacterial enteritis (29 cases), followed by Shigella spp. - 26.2\% (16 cases) and Campylobacter - $11.47 \%$ (7 cases).

The most common cause of enteritis with confirmed infectious etiology in adults was Salmonella spp., with a percentage of $47.9 \%$ (23 cases), followed by Clostridium spp. with $29.1 \%$ (14 cases) and Shigella spp. with $16.6 \%$ (8 cases). The strains of Salmonella spp. that had been isolated from adults were $100 \%$ sensitive to: quinolones, cephalosporins, imipenem, gentamicin, but $26 \%$ (6 cases) were resistant to tetracycline.

\section{Conclusion}

Rotavirus is the most common cause of hospitalized enteritis in children. Both in children over 2 years and adults the most common pathogen in bacterial enteritis is Salmonella. A great percentage of post-antibiotic AD in adults is caused by Clostridium spp.

\section{Authors' details}

${ }^{1}$ Clinical Infectious Diseases Hospital of Constanța, Romania. ${ }^{2}$ Faculty of Medicine, "Ovidius" University, Constanța, Romania.

Published: 15 October 2014

doi:10.1186/1471-2334-14-S7-027

Cite this article as: Dumitrescu et al:: Etiology of acute diarrhea in patients requiring hospitalization in Clinical Infectious Diseases Hospital - Constanța. BMC Infectious Diseases 2014 14(Suppl 7):O27. 